\title{
Not so lonely: The LBV binary HR Car
}

\author{
T. Rivinius ${ }^{1}$, H. M. J. Boffin ${ }^{1,2}$, A. Mérand ${ }^{1,2}$, A. Mehner ${ }^{1}$, \\ J.-B. LeBouquin ${ }^{3}$, D. Pourbaix ${ }^{4}$, W.-J. de Wit ${ }^{1}$ and C. Martayan ${ }^{1}$ \\ ${ }^{1}$ ESO - European Organisation for Astronomical Research in the Southern Hemisphere, Chile \\ ${ }^{2}$ ESO, Karl-Schwarzschild-Str. 2, 85748 Garching, Germany \\ ${ }^{3}$ Institut de Planétologie et d'Astrophysique de Grenoble, 38041 Grenoble, France \\ ${ }^{4}$ Institut d'Astronomie et d'Astrophysique, Université Libre de Bruxelles (ULB), Belgium
}

\begin{abstract}
Luminous Blue Variables (LBVs) are a brief phase in the evolution of massive stars, but a very important one. The giant eruptions remain enigmatic, but the discovery of the flagship LBV $\eta$ Car to be a five-year highly eccentric binary put focus on possible binarity induced mechanism for these outbursts, and prompted binarity searches among LBVs. While several wide LBV binaries were identified, HR Car is the first system found to be similar to $\eta$ Car, i.e., relatively close \& eccentric.
\end{abstract}

Keywords. binaries: general, stars: individual: HR Car, stars: variables: other

After an interferometric snapshot campaign with AMBER at the VLTI in 2013/14, HR Car was suspected to be a close binary. A follow-up campaign with PIONIER, a 4-beam interferometric instrument working in the $H$-band continuum confirmed the binarity and allowed initial constraints on the orbit. Analysis of the obtained PIONIER data firmly establishes the presence of two different sources with a contrast ratio of about 1:6 to 1:9. The components are marginally resolved (P: $0.38 \pm 0.08$ mas, $\mathrm{S}: 0.85 \pm 0.2 \mathrm{mas}$ ). The best orbital fit parameters from current data are shown in Table 1, but since the first orbit is not yet complete, the parameters are preliminary: While shorter orbits are excluded, much longer ones remain possible. See Boffin et al.(2016) for a complete discussion of the observations and fitting process.

At the distance of $5.4 \pm 0.4 \mathrm{kpc}$, the primary's size would be $220 \pm 60 \mathrm{R}_{\odot}$, too large for the LBV in minimum, but acceptable if wind/nebulosity is taken into account. The secondary, much larger than the primary, could be either a red supergiant, or disturbed from equilibrium by a previous orbital passage: HR Car was in a maximum until 2001.

During LBV minimum, the periastron distance is about three times larger than the sum of radii. The last periastron was about 2014.5, around the times of AMBER observations, which show some sign of wind-wind interaction. During LBV maximum, though, the secondary may even pass through the extended outer layers of the primary, a scenario that may lead to clearly observable effects and new insights into $\eta$ Car type binarity.

Table 1. Parameters of the best orbit that fits the PIONIER observations.

\begin{tabular}{lc|lc|lc}
\hline$\Omega$ (deg.) & $46.9 \pm 0.6$ & $P$ (days) & $4557.5 \pm 21.0$ & $T_{0}$ (MJD) & $56990.6 \pm 16.0$ \\
$a$ (mas) & $3.324 \pm 0.026$ & $e$ & $0.4 \pm 0.2$ & $i$ (deg.) & $119.2 \pm 0.7$ \\
$\omega$ (deg.) & $201.9 \pm 2.1$ & Flux ratio $H$-band & $1: 6$ to $1: 9$ & & \\
\hline
\end{tabular}

\section{Reference}

Boffin, H. M. J., Rivinius, T., Mérand, A., et al. 2016, A\&A, 593, A90 